\title{
Simultaneous blind separation and clustering of coactivated EEG/MEG sources for analyzing spontaneous brain activity
}

\author{
Jun-ichiro Hirayama $^{1}$, Takeshi Ogawa ${ }^{1}$ and Aapo Hyvärinen ${ }^{2,1}$
}

\begin{abstract}
Analysis of the dynamics (non-stationarity) of functional connectivity patterns has recently received a lot of attention in the neuroimaging community. Most analysis has been using functional magnetic resonance imaging (fMRI), partly due to the inherent technical complexity of the electro- or magnetoencephalography (EEG/MEG) signals, but EEG/MEG holds great promise in analyzing fast changes in connectivity. Here, we propose a method for dynamic connectivity analysis of EEG/MEG, combining blind source separation with dynamic connectivity analysis in a single probabilistic model. Blind source separation is extremely useful for interpretation of the connectivity changes, and also enables rejection of artifacts. Dynamic connectivity analysis is performed by clustering the coactivation patterns of separated sources by modeling their variances. Experiments on resting-state EEG show that the obtained clusters correlate with physiologically meaningful quantities.
\end{abstract}

\section{INTRODUCTION}

Typically, the statistical dependence of neural signals in different brain regions (i.e., functional connectivity [5]) dynamically changes over time. These changes may reflect critical aspects of information processing related to cognitive or behavioral functions [6]. Analysis of such dynamic functional connectivity, especially in the case where the brain activity spontaneously fluctuates during resting state, has been gaining increasing attention in both neuroimaging [6], [16], [9] and neuroengineering [12]. A key technical challenge here is to explore the statistical regularities that intrinsically exists in spontaneous brain activity, which needs well-designed exploratory/unsupervised analysis methods.

One of the most fundamental methods used so far for analyzing dynamic functional connectivity is clustering. For example, previous studies on resting-state functional magnetic resonance imaging (fMRI) have used the well-known $k$ means clustering to categorize the signals' coactivations [10] or correlations [1] across the whole brain into a few discrete states, as well as to find unique patterns (cluster centroids) associated with these states. Both the estimated states and the patterns obtained provide a simple and intuitive way of analyzing the data. A similar approach would also be

\footnotetext{
*This research was supported by a contract with the Ministry of Internal Affairs and Communications entitled, 'Novel and innovative R\&D making use of brain structures' and by JSPS KAKENHI Grant Number 25730155.

${ }^{1}$ J. Hirayama, T. Ogawa and A. Hyvärinen are with Cognitive Mechanisms Laboratories, Advanced Telecommunications Research Institute International (ATR), 2-2-2 Hikaridai Seika-cho, Sorakugun, Kyoto, 619-0288 Japan $\{$ hirayama, t.ogawa $\}$ at atr.jp

${ }^{2}$ A. Hyvärinen is with Department of Computer Science and HIIT, University of Helsinki, Gustaf Hällströmin katu 2b, 00560 Helsinki, Finland aapo.hyvarinen at helsinki.fi
}

very useful for analyzing spontaneous electroencephalography (EEG) and magnetoencephalography (MEG), especially for characterizing the brain states that change relatively fast. This is also highly relevant for advanced brain-computer interfacing (BCI) technologies.

Clustering has previously been used directly on the EEG/MEG sensor space, e.g., in studies on EEG microstates [4] or on estimating subjects' vigilance levels from EEG [15]. However, a more promising application of "connectivity clustering" to EEG/MEG can be obtained by first separating the neural sources from the observed mixtures (sensor measurements) prior to clustering, either by blind source separation (BSS) methods or by solving electromagnetic inverse problems. Blind source separation has the advantage of greatly simplifying the interpretation of the results by decomposing the data into components, and the inverse problem can be solved to localize the obtained components afterwards.

The problem is that solving the two different problems one-by-one (stage-wise), i.e., doing first source separation and then clustering, is "neither a principled nor an optimal solution to the overall problem" [12]. Hence, a unified approach would be desirable for improving the quality of clustering/BSS as well as the interpretability of result.

Here, we propose a novel method that combines the BSS and connectivity clustering of EEG/MEG sources in a principled manner. Our basic idea is to use a finite mixture model of the sources that exhibit state-dependent coactivation patterns, characterized by their amplitude envelopes. The source model is then integrated with a model of complexvalued BSS. Due to its simplicity, the model is tractable, and the entire model can be simultaneously estimated (optimized) with a unified objective.

\section{PROPOSED METHOD}

\section{A. Complex-valued BSS based on Hilbert transform}

Consider a basic set-up for blind separation of EEG/MEG sources. Let $\boldsymbol{x}(t)=\left(x_{1}(t), x_{2}(t), \ldots, x_{d}(t)\right)^{\top} \in \mathbb{R}^{d}$ be an EEG/MEG signal with discrete time index $t=1,2, \ldots, N$, measured in $d$ sensor channels and have been preprocessed appropriately. Each $x_{j}(t)$ is then assumed to be a linear instantaneous mixture of $d$ sources, that is,

$$
\boldsymbol{x}(t)=\mathbf{A} \boldsymbol{s}(t)
$$

where $s(t)=\left(s_{1}(t), s_{2}(t), \ldots, s_{d}(t)\right)^{\top} \in \mathbb{R}^{d}$ denotes the source signal. The mixing matrix $\mathbf{A} \in \mathbb{R}^{d \times d}$ is assumed to be non-singular, so that the demixing matrix $\mathbf{W}:=\mathbf{A}^{-1}$ exists. Every $s_{j}(t)$ is further assumed to have zero mean, 
without loss of generality, as we always subtract the sample mean from the sensor signals. Note that the numbers of sensors and sources are assumed to be equal for mathematical simplicity. However, the same setting is directly available when more sensors than sources exist, e.g., with the sensor dimensionality reduced by principal component analysis.

Instead of directly solving the BSS problem above, we convert the problem to a complex-valued BSS, modeled similarly as

$$
\widetilde{\boldsymbol{x}}(t)=\mathbf{A} \widetilde{\boldsymbol{s}}(t),
$$

where $\widetilde{\boldsymbol{x}}(t) \in \mathbb{C}^{d}$ and $\widetilde{\boldsymbol{s}}(t) \in \mathbb{C}^{d}$ are the complex sensor and source signals, respectively. Specifically, we use the complex analytic signals defined in each sensor and source channel as $\widetilde{x}_{j}(t):=x_{j}(t)+i \mathcal{H}\left[x_{j}\right](t)$ and $\widetilde{s}_{j}(t):=s_{j}(t)+i \mathcal{H}\left[s_{j}\right](t)$, respectively, where $i$ denotes the imaginary unit, and $\mathcal{H}[f]$ denotes the Hilbert transform of a signal $f(t)$. Note that the mixing matrix $\mathbf{A}$ in Eq. (2) is identical to the original one in Eq. (1) because of the linearity of the Hilbert transform; it thus can be interpreted directly in the original sensor space.

The problem is now to estimate both $\mathbf{A}$ and $\widetilde{\boldsymbol{s}}(t)$ from $\widetilde{\boldsymbol{x}}(t)$ that can be computed directly from the original $\boldsymbol{x}(t)^{1}$, which requires suitable assumptions (a model) about the sources. The fundamental motivation for this Hilbert transformation is that the analytic source $\widetilde{s}_{j}(t)$ gives the (instantaneous) amplitude of $s_{j}(t)$ simply as its modulus [14], i.e., $\alpha_{j}(t):=$ $\left|\widetilde{s}_{j}(t)\right|$, which greatly simplifies the development below.

\section{B. Latent coactivity mixture model (LCMM)}

We introduce a novel statistical model for the $\widetilde{s}_{j}(t)$ in Eq. (2) to perform clustering of source connectivities and the complex-valued BSS in a unified manner. A fundamental assumption here is that the system generating the data can be in a finite number of different states (indexed by $k=1,2, \ldots, K)$, reflected in different levels of the source amplitudes.

The proposed latent coactivity mixture model (LCMM) defines the following generative process of the complex sensor signal $\widetilde{\boldsymbol{x}}(t)$ for each $t=1,2, \ldots, N$, simply treated as i.i.d. in time:

1) State $k$ occurs with probability $\eta_{k}$

2) Sources $\widetilde{\boldsymbol{s}}(t)$ are generated as $\widetilde{\mathcal{T}}\left(\widetilde{\boldsymbol{s}} ; \operatorname{diag}\left(\boldsymbol{b}_{k}\right), \nu\right)$, with their variances depending on $k$

3) Sensor signal $\widetilde{\boldsymbol{x}}(t)$ is given by Eq. (2)

where $\tilde{\mathcal{T}}(\cdot ; \boldsymbol{\Sigma}, \nu)$ denotes the probability density function (pdf) of the multivariate Student-t distribution for complex circular random vectors [14], parameterized by the scatter matrix $\Sigma$ and the degrees of freedom $\nu$, given by,

$$
\widetilde{\mathcal{T}}(\widetilde{\boldsymbol{s}} ; \boldsymbol{\Sigma}, \nu)=\frac{2^{d} \Gamma\left(d+\frac{\nu}{2}\right)}{\Gamma\left(\frac{\nu}{2}\right)(\nu \pi)^{d}|\boldsymbol{\Sigma}|}\left(1+\frac{2}{\nu} \widetilde{\boldsymbol{s}}^{\top} \boldsymbol{\Sigma}^{-1} \widetilde{\boldsymbol{s}}\right)^{-d-\frac{\nu}{2}},
$$

where $\Gamma(\cdot)$ denotes the Gamma function. The circularity implies that the (instantaneous) phase is distributed uniformly and independently of the amplitude. Roughly speaking, $\boldsymbol{b}_{k}$

\footnotetext{
${ }^{1}$ For accurate computation of the Hilbert transform, we here focus on band-limited signals $\boldsymbol{x}(t)$ after preprocessing.
}

sets expected levels of the instantaneous powers (i.e., squared amplitude) of the sources in different states, and is here termed the coactivation patterns as in [10].

The Student-t model of the complex sources, Eq. (3), has desirable properties for connectivity clustering of EEG/MEG sources. First, as is well-known, it has a robustness property for the estimation of (co)variances, which is proportional to $\Sigma$ [11], assuming the covariances exist. Second, the coactivated amplitudes have homogeneous correlations, which serves as a parsimonious model of connectivity within each state: The conditional correlation coefficient, given the state $k$, between any two amplitudes $\alpha_{j}$ and $\alpha_{j^{\prime}}\left(j \neq j^{\prime}\right)$ is a positive constant that depends only on $\nu$ (assuming neither of the variances $b_{j k}, b_{j^{\prime} k}$ is zero; the proof is omitted due to the lack of space).

The LCMM now gives the pdf in the complex sensor space as $p(\widetilde{\boldsymbol{x}})=\sum_{k=1}^{K} \eta_{k} \widetilde{\mathcal{T}}\left(\widetilde{\boldsymbol{x}} ; \mathbf{A}^{\top} \operatorname{diag}\left(\boldsymbol{b}_{k}\right) \mathbf{A}, \nu\right)$; it can be conveniently expressed using $\mathbf{W}=\mathbf{A}^{-1}$ as

$$
p(\widetilde{\boldsymbol{x}})=|\operatorname{det} \mathbf{W}| \sum_{k=1}^{K} \eta_{k} \widetilde{\mathcal{T}}\left(\mathbf{W} \widetilde{\boldsymbol{x}} ; \operatorname{diag}\left(\boldsymbol{b}_{k}\right), \nu\right) .
$$

The form of Eq. (4) is similar to the standard model of independent component analysis (ICA) [7], while the sources are now not independent of each other. Compared to other hierarchical extensions of ICA with dependent sources (e.g., [8]), the LCMM is tractable and fast to learn, because it has only one discrete state but not multiple continuous ones that may complicate the estimation.

\section{Parameter estimation}

The parameters of the LCMM are $\{\mathbf{W}, \mathbf{B}, \boldsymbol{\eta}\}$, where $\mathbf{B}:=\left(\boldsymbol{b}_{1}, \ldots, \boldsymbol{b}_{K}\right)$ collects the coactivation patterns, and the vector $\boldsymbol{\eta}$ collects the state probabilities $\eta_{k}$ 's. The parameters can be easily estimated by the maximum likelihood method. The degrees of freedom $\nu$ could also be estimated from data, while in practice we fix it to as a pre-set constant (we used $\nu=2$ below) as it does not greatly affect the solutions. We specifically used a quasi-Newton method $^{2}$ in our experiments below to minimize the negative log-likelihood, i.e., $(1 / N) \sum_{t=1}^{N}\{-\ln p(\boldsymbol{x}(t))\}+$ const., where we used a reparameterization [13] of $\eta_{k}=\exp \left(\eta_{k}^{\prime}\right) / \sum_{k^{\prime}=1}^{K} \exp \left(\eta_{k^{\prime}}^{\prime}\right)$ with $\eta_{k}^{\prime}$ 's, so that $\eta_{k}$ automatically satisfied the constraints, $\eta_{k} \geq 0$ and $\sum_{k=1}^{K} \eta_{k}=1$, to be a probability distribution.

After obtaining the estimates of the model parameters, the (real-valued) sources are separated by $\boldsymbol{s}(t)=\mathbf{W} \boldsymbol{x}(t)$ for any given $\boldsymbol{x}(t)$. The states are then inferred by first Hilbert transforming a time segment of $\boldsymbol{x}(t)$ into corresponding complex signal $\widetilde{\boldsymbol{x}}(t)$ and then computing the posterior probability of the state, with the prior probabilities $\eta_{k}$ set appropriately (e.g., to uniform probabilities). The maximum a posteriori (MAP) estimate of the state is then given by taking the state that maximizes this posterior, which gives the final result of clustering.

\footnotetext{
${ }^{2}$ We used a Matlab implementation of the limited-memory BFGS by Mark Schmidt (http://www.di.ens.fr/ mschmidt/Software/minFunc.html)
} 


\section{EXPERIMENTAL RESULTS}

Here, we first validate the proposed method in a simulation experiment and then demonstrate it with a real EEG dataset.

\section{A. Comparison with stagewise approaches}

We start by simulated EEG (or MEG) sources that are rhythmic with their amplitudes jointly changing. The six $(d=6)$ sources $s_{j}(t)$ were created as follows. First, we applied a band-pass filter $(9.5-10.5 \mathrm{~Hz})$ to Gaussian white signals sampled virtually at $75 \mathrm{~Hz}$ to simulate alpha waves. Then, they were amplitude-modulated block-wise in every 2 -second window (150 samples) to show state-dependent coactivation patterns; i.e., the $j$-th signal was multiplied by $\sqrt{b_{j k}}$, with the state $k$ randomly chosen for each block with uniform probability $(k=1,2,3)$. Each entry of $\boldsymbol{b}_{k}$ was independently sampled from a standard Gaussian but was set to zero if negative, and this was repeated until when at least two entries satisfied $b_{j k} \geq 0.05$. The sources with non-zero $b_{j k}$ 's were then actually coactivated, while very small activity levels were avoided with this procedure. Finally, the amplitudes of these coactivated sources were globally modulated by a common modulatory signal (a noisy sinusoidal signal), and Gaussian white noise was added (SNR: $30 \mathrm{~dB}$ ).

The goal of this simulation was to show that the new unified approach, i.e., simultaneous optimization of the source separation and clustering, improves the quality of solution in both problems. We thus made a comparison between this simultaneous method and two other methods with stagewise solutions, namely, stagewise and k-means: The latter two methods first used the complex-valued FastICA [3] for separating the complex sources $\widetilde{\boldsymbol{s}}(t)$; then, stagewise learned the mixture of Student-t model of LCMM directly on the separated sources, while $k$-means performed the standard $k$ means clustering on the $\log$-amplitudes $\log \left|\widetilde{s}_{j}(t)\right|$ (where the mean log-amplitude over channels was subtracted at every $t$ to compensate for the global modulation).

Figure 1 compares the performance of these three methods in clustering (left) and in source separation (right), when the number of clusters was assumed to be known $(K=3)$. Here, the six sources $s_{j}(t)$ were linearly mixed into the sensor signal $\boldsymbol{x}(t)$ with a mixing matrix $\mathbf{A}$ generated randomly in each run, and we conducted 50 runs with each specific sample size $N$ shown in the figure. Note that in the right panel, "LCMM" corresponds to simultaneous and "ICA" to both stagewise and k-means. As is clearly seen, the proposed simultaneous approach improved the relevant performance measures, especially when the sample size was relatively small. The relative performance was similar even when the number of $K$ was misspecified, which is shown in Fig. 2.

\section{B. Application to resting-state EEG analysis}

We next demonstrate the proposed method of connectivity clustering with real resting-state EEG data. The clustering is validated using further task-based data and task labels.

Our EEG experiment consisted of two resting-state (RS) sessions, including six task sessions between the RS sessions.
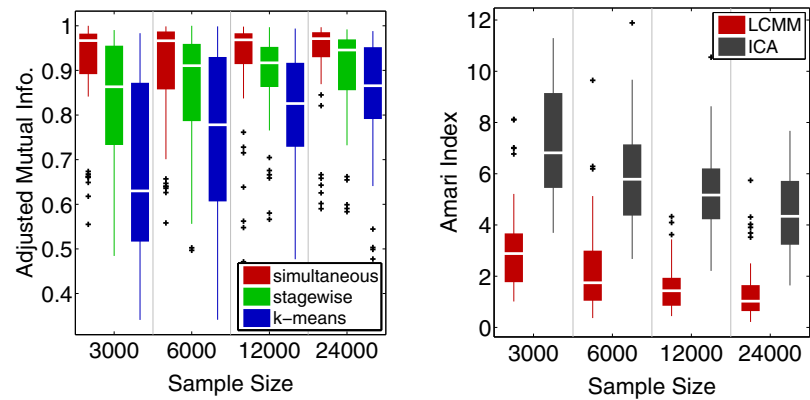

Fig. 1. Simulated data (with true $K=3$ ): Performance in clustering (left) and source separation (right) with different sample sizes, measured respectively by the Adjusted Mutual Information [17] (scaled between 0 [completely random] and 1 [perfect]) and the Amari Index [2] (which equals zero if the true $\mathbf{A}$ is recovered up to the permutation and scaling of the columns). Each box and whisker shows the interquartile range (with the median) and the entire data range, respectively, of the 50 runs, excluding some outliers indicated by + . See text for the details of the legends. (a) $K=2$

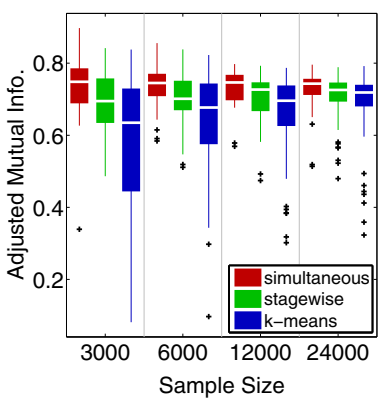

(b) $K=6$

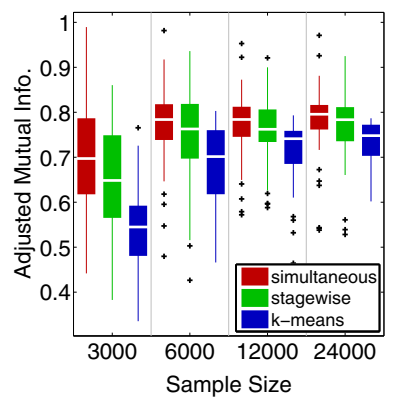

Fig. 2. Simulated data (with misspecified $K$ 's): Clustering performance shown in the same format in Fig. 1 (left). See also the caption of Fig. 1. The true number of clusters was always three.

In each RS session (5 minutes), a single healthy subject was instructed to relax without thinking of anything particular and without sleeping, while focusing on a fixation point at the center of a screen. In each task session, the subject was instructed to perform a cued motor imagery/non-imagery. The task sessions contained repeated trials of three different actions; namely, the subject was asked in a random order to either 1) Left: covertly imagine a left-hand movement, 2) Right: covertly imagine a right-hand movement, or 3) Idle: not imagine hand movements; each of these for 3 seconds after a visually-cued onset.

The EEG data (Active-Two amplifier, $64 \mathrm{CH}$ active electrodes, BioSemi, the Netherlands) was acquired at a sampling frequency of $256 \mathrm{~Hz}$, band-pass filtered off-line to $1-50 \mathrm{~Hz}$ (4th-order butterworth, zero phase), and re-referenced to the common average. A part of the RS data and some trials in the task data were rejected due to gross contamination. Typical ocular, cardiac and muscle artifacts were also identified and removed by FastICA with visual inspection and frequency analysis. The RS data eventually contained a total of $N=$ 97,536 samples $\boldsymbol{x}(t)$. The task data contained 65,61 and 66 trials for Left, Right and Idle.

In the analysis, all the parameters of the LCMM were learned from the RS data alone. The task data were used 


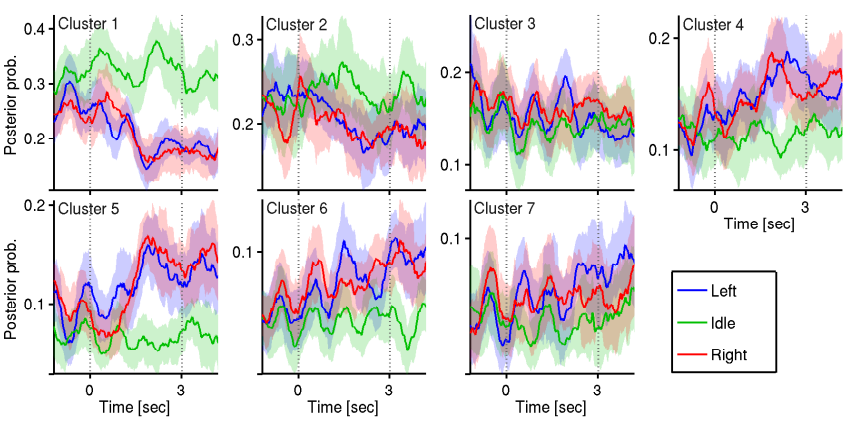

Fig. 3. Real EEG analysis: Averaged time-courses of the posterior state (cluster) probabilities during the task trials of cued motor imagery or nonimagery, with the LCMM trained on other resting-state EEG data. Only the seven most frequent clusters (out of ten) are shown due to the limitation of space. The three plots in different colors show the posterior values of each cluster, averaged over all trials of the three different actions: Left (motor imagery on left hand; blue), Right (on right hand; red), and Idle (no imagery; green). The solid lines indicate their moving-average with time windows of 0.5 [sec], and the shaded intervals indicate the standard deviation in each moving window. The horizontal time axis is relative to the cued onset, where the two vertical lines indicate the time period over which the subject was instructed to keep the imagery or non-imagery.

only to post-hoc investigate the physiological validity of the learned model. The frequency band of interest was set to 13$30 \mathrm{~Hz}$ (Beta band; filtered with 4th-order butterworth, zerophase). The number of clusters was set to 10 , which was selected for ease of visualization, while similar clusters were often found at least for $20,30, \ldots, 60$ (not shown here).

Figure 3 shows the averaged time-courses of the posterior state probability, based on the task data and a uniform prior $\eta_{k}$. Among the seven clusters displayed here, the cluster \#1, and possibly \#2, seem to be activated frequently in Idle, while activated less frequently (or deactivated frequently) in Left or Right with a latency of about 1.5 seconds after the task onset. In contrast, the clusters \#4, \#5, and \#6 seem to become activated frequently with a similar latency in Left or Right, but not in Idle. No clusters seemed to be specifically correlated with either Left or Right. Examples of scalp topographies of coactivated sources, associated with the clusters \#1 and \#5, are shown in Fig. 4. The result could be interpreted as suppression of spatially-distributed network of the sources (\#1), while activation of more spatially-focused one (\#5), during motor imagery. Note that the patterns were found in spontaneous EEG data. Thus, even the spontaneous EEG sources intrinsically contain task-relevant coactivation patterns, which may be modeled and learned by unsupervised methods such as LCMM.

\section{CONCLUSION}

We proposed a method combining blind source separation and clustering to analyze the dynamics of functional connectivity in EEG/MEG. The method is based on estimation of a single probabilistic model, thus avoiding the unoptimal separation of the two stages of analysis. Unlike related two-stage models, the model is tractable and inference computationally simple. Results on resting-state EEG indicate that the clusters learned are related to meaningful brain states.

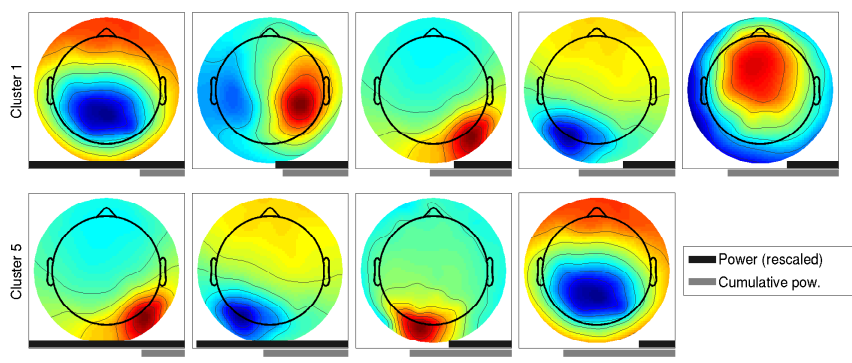

Fig. 4. Real EEG analysis: Scalp topographies of coactivated EEG sources found by LCMM. The top and bottom rows correspond to the clusters \#1 and \#5 in Fig. 3, activated more frequently in Idle and in Left/Right, respectively. The panels in each row show the scalp topographies (each given by a column of $\mathbf{A}$ ) of the sources that activate relatively strongly in the cluster, i.e., have relatively large $b_{j k}$ (expected powers). The relative strength is indicated by the width of the black bar at the bottom of each panel. The gray bars indicate their cumulative score such that the full panel width indicates the total sum, normalized to 1 . Only the topographies that keep more than the score of 0.7 are displayed here.

\section{REFERENCES}

[1] E. A. Allen et al. Tracking whole-brain connectivity dynamics in the resting state. Cerebral Cortex, 24(3):663-676, 2014.

[2] S. Amari, A. Cichoki, and H. H. Yang. A new learning algorithm for blind signal separation. In Advances in Neural Information Processing Systems, volume 8, 1996.

[3] E. Bingham and A. Hyvärinen. A fast fixed-point algorithm for independent component analysis of complex valued signals. Int. J. Neural Syst., 10(1):1-8, 2000.

[4] Michel CM Britz J, Van De Ville D. BOLD correlates of EEG topography reveal rapid resting-state network dynamics. Neuroimage, 52:1162-1170, 2010.

[5] K. J. Friston. Functional and effective connectivity in neuroimaging: A synthesis. Hum Brain Mapping, 2:56-78, 1994.

[6] R. M. Hutchison et al. Dynamic functional connectivity: Promise, issues, and interpretations. NeuroImage, 80(360-378), 2013.

[7] A. Hyvärinen, J. Karhunen, and E. Oja. Independent Component Analysis. John Wiley \& Sons, 2001

[8] Y. Karklin and M. S. Lewicki. A hierarchical Bayesian model for learning nonlinear statistical regularities in nonstationary natural signals. Neural Comput., 17:397-423, 2005.

[9] N. Leonardi et al. Principal components of functional connectivity: A new approach to study dynamic brain connectivity during rest NeuroImage, 83:937-950, 2013.

[10] X. Liu, C. Chang, and J. H. Duyn. Decomposition of spontaneous brain activity into distinct fMRI co-activation patterns. Frontiers in systems neuroscience, 7:101, 2013.

[11] M. Mahot et al. Asymptotic properties of robust complex covariance matrix estimates. IEEE Transactions on Signal Processing, 61(13):3348-3356, 2013

[12] S. Makeig et al. Evolving signal processing for braincomputer interfaces. Proceedings of the IEEE, 100(Centennial-Issue):15671584,2012

[13] R. Salakhutdinov, S. T. Roweis, and Z. Ghahramani. Optimization with $\mathrm{em}$ and expectation-conjugate-gradient. In International Conference on Machine Learning (ICML) 20, pages 672-679, 2003.

[14] P. J. Schreier and L. L. Scharf. Statistical Signal Processing of Complex-Valued Data. Cambridge University Press, 2010.

[15] L. C. Shi and B. L. Lu. Dynamic clustering for vigilance analysis based on EEG. In Conf. Proc. IEEE Eng Med Biol Soc, 2008.

[16] S. M. Smith et al. Temporally-independent functional modes of spontaneous brain activity. Proc. Natl. Acad. Sci. USA, 109(8):31313136,2012

[17] N. X. Vinh, J. Epps, and J. Bailey. Information theoretic measures for clusterings comparison: Variants, properties, normalization and correction for chance. Journal of Machine Learning Research, 11:2837$2854,2010$. 